\title{
Novel Computerized Approaches to Investigating Pharmacological Activities
}

\author{
Nwankwo Norbert ${ }^{1,}$, , Godwin Molokwu ${ }^{2}$, Ngozika Njoku ${ }^{3}$ \\ ${ }^{1}$ Department of Clinical Pharmacy, Faculty of Pharmacy, University of Port Harcourt, Port Harcourt, Nigeria \\ ${ }^{2}$ Department of Pharmacology, Faculty of Pharmacy, Madonna University, Elele, Nigeria \\ ${ }^{3}$ Ngozika Njoku, Nova Psychiatric Services, Quincy, USA \\ Email address: \\ nobatn@gmail.com (N. Nwankwo),dchmolos@yahoo.com (G. Molokwu),ngozikanjoku@yahoo.com (N. Njoku)
}

\section{To cite this article:}

Nwankwo Norbert, Godwin Molokwu, Ngozika Njoku. Novel Computerized Approaches to Investigating Pharmacological Activities. Computational Biology and Bioinformatics. Vol. 3, No. 4, 2015, pp. 52-64. doi: 10.11648/j.cbb.20150304.12

\begin{abstract}
Complementing clinical assessments using computerized procedures with the view of completely disengaging from clinical procedures may become inevitable in future. Huge biological and pharmacological data (such as sequences) are churned out daily such that it is becoming difficult to process them without the aid of computers. Computerized approaches including Digital Signal Processing (DSP)-based Bioinformatics procedures like Informational Spectrum Method (ISM) are rational techniques that need be incorporated into pharmacological studies. By means of ISM and one biological parameter or Amino Acid Scale (AAS), we have preliminarily shown how pharmacological activities can be decoded using computerized techniques. However, for effective engagement of ISM, some basic information must be made available and engaged. Firstly, the sequence information comprising of the consensus sequences and all the mutations involved must be assembled and engaged. Pharmacological activities (e.g. drug resistance) are known to be expressed in the genes/proteins (e.g.MDR1 and MDR2, pfdr1, etc). Secondly, biological parameters must be identified and engaged. This calls for good knowledge of the drugs' mechanisms of action at the atomic level. This is because it has been identified that, at one point mutation; more than one biological parameter may be involved. To obtain the entirety of pharmacological activities exhibited therefore, aggregation of the contributions from each mutation and parameter is needed.We have then unveiled and compared the pharmacological activities of anti-retroviral agents (Enfuvirtide and Sifuvirtide), and potencies of Malaria vaccine candidates, peptides P18 and P32 (Innocentive Challenge Winning Solver Award, ID: 9933477), etc. A biomedical device called Computer-Aided Drug Resistance Calculator (Patent Application filed in 2014) is developed using this novel computerized approach. The device will rationally help assess a pharmacological property (drug resistance). Other researchers have recorded in-silico pharmacological assessments. Clinically and computationally derived outcomes are found to correlate. We therefore propose that these computerized approaches be engaged in deciphering pharmacological activities where sequence information and biological parameters are available. These approaches are rational. They also present pharmacological findings in numerical terms. In this era of rational, computerized, informatics- and robotics-based procedures, these approaches are envisaged to transform pharmacological investigation procedures especially now that pharmacological activities could be deciphered from their protein sequences or those of their protein targets and the genes/proteins expressing them. The procedures engaged in this study are expected to be embodied into Pharmaco-informatics program.
\end{abstract}

Keywords: Amino Acid Scale, Digital Signal Processing, Informational Spectrum Method

\section{Introduction}

It is evident that in the nearest future, complete disengagement from clinical approaches to assessing pharmacological properties of therapeutic interventions may be unavoidable. Currently, clinical assessments are being supported by computational approaches. This is because computational approaches are resource and time saving as they are not labor-intensive [1, 2]. Such computational approaches include the Digital Signal Processing (DSP)-based Bioinformatics procedures like Informational Spectrum Method (ISM) [3-5] and Resonant Recognition 
Method (RRM) [6].

We have preliminarily engaged ISM and one biological parameter, Amino Acid Scale (AAS), to show how we can extract pharmacological activities from sequence information [7-9]. AASs are defined as the numerical values representing the level of participation of the each of the 20 essential amino acids that constitute protein in various interactions [7]. Some of the AASs are deposited at the GeomeNet Database Resources (www.genome.jp/aaindex) [10]. A number of the pharmacological activities have computationally been obtained by analyzing their sequence information using ISM and one appropriate AAS. They include resistances offered by five classes of anti-retroviral agents on their target. Fusion/Entry Inhibitors (Enfuvirtide) was investigated using ROBB760104 [9-11] while Nucleotide Reverse Transcriptase Inhibitors (Lamivudine) engaged BEGF750102 [8-10]. WILM950103 [8-10, 12] was employed in the study of Protease Inhibitors (Darunavir). Other antiretroviral agent studied using ISM and a biological parameter includes Maturation Inhibitors (Bevirimat (BVM)). In this case, EIIP was utilized [3- 6, 10]. Integrase Enzyme Inhibitors (Raltegravir) was examined by means of LIFS790103 [5, 6, 10]. The decreases in the levels of various interactions demonstrated by the mutated protein sequences of the drug targets is a representation of resistances acquired by the viruses as they were exposed to the drugs. This procedure has helped develop a biomedical device called Computer-Aided Drug Resistance Calculator. This device originated from [9] and was proposed in [8]. A functional Computer-Aided Drug Resistance Calculator was later developed and used to assess drug resistance acquired by the HIV Protease enzyme exposed to Amprenavir, which has all point mutations and parameters derived (Patent Application filed in 2014). It is expected to rationally address drug resistance assessments.

In order to engage ISM to uncover pharmacological activities, some fundamental information must be presented and engaged. All biological parameters (Amino Acid Scales, AASs) and sequence information involved in the interactions between the pharmacological agents and their targets must be employed. This is because, it has been noted that several biological parameters are involved at one point mutation [14] Therefore, summation of all the pharmacological input from all mutations and biological parameters is required to obtain the entirety of the pharmacological activities exhibited. Currently, over 575 biological parameters have been identified [14].

Sequence information implies the consensus sequences and all the mutations involved. It is important to note that the nature of the pharmacological agent (alkaloids, quinone, anthraquinone, flavonoids, terpenes, steroids, etc) is not necessary. This is because every pharmacological activity is expressed or encoded in the genes or proteins [15]. Pharmacological agents therefore carry as much information as the genes or proteins expressing them. For example, Multi-Drug Resistance 1 and 2 (MDR1 and MDR2) are known to encode resistances of a wide range of anti-microbial agents [16]. Similarly, the resistance offered by Plasmodium falciparum, the causative agent for Malaria over a wide range of drugs is expressed in $p f d r 1$. This may explain why in the human proteome, there are 20,000-25,000 protein-coding genes from which over 30,000 distinctive protein sequences have been uncovered from [17]. Identification of the genes or proteins associated with pharmacological activities may remain therefore ongoing [18]. Analysis of MDR1 and MDR11 or pfdr1 can then proffer the required information on the resistance or anti-Malarial profiles of these large numbers of anti-microbial or anti-Malarial agents.

Engaging all the biological parameters and sequence information involved, we have computationally investigated the pharmacological activities of two anti-retroviral agents, Enfuvirtide and Sifuvirtide using ISM, and further compared their potencies $[9,19]$. Sifuvirtide, a product of biomedical modification of CHR motif including Enfuvirtide was found to be more efficacious. Increased efficacy was achieved through Helicity- and Hydrophobicity-oriented biomedical modifications [9, 20]. Therefore, Helicity-, EIIP- and Hydrophobicity-oriented biological parameters were obtained from [10] and utilized. Our ISM-based findings [9, 19] show correlation with the clinical outcomes. Sifuvirtide, adjudged superior to Enfuvirtide clinically [20] recorded greater interaction $(85.42 \%)$ than Enfuvirtide (72.33\%).

Equally, we investigated potencies of two peptidic vaccine candidates, P18 and P32 using these computerized approaches $[9,19]$. P18 and P32 were found to interact with their target protein, Glycosaminoglycan (GAG) using negatively charged carboxyl group [21, 22]. Hence, Charge Transfer-based biological parameters and EIIP involved were retrieved from [10] and used. Both peptides offered 100\% in bio-recognition and bio-attachment (by means of EIIP) showing tendency to appropriately identify the disease to immunize against. P32 has overall greater potency (95.15\%) than P18 (93.40\%). Part of this study provided a computational elucidation to "Assessing Vaccine Potency" that won Innocentive Challenge Winning Solver Award (ID: 9933477).

Single point mutant (nDARC Y41F) of a 35 amino acid length of a Plasmodial host protein from the Duffy Antigen Receptor Chemokines is clinically recognized to abrogate the blockage of inhibitory activity of $\mathrm{nDARC}$ on the Plasmodial protein called Duffy Binding Protein (DBP) [22]. This was computationally evaluated [9]. Our results demonstrated correlation with the clinical outcomes as the computed inhibitory activities of the nDARC $(96.88 \%)$ is completely wiped off by the mutation nDARC Y41F (100\%), signifying total recovery (abrogation of inhibition). Major mechanism of action is observed to through binding as only the EIIP showed changes in activity, nDARC (84.4\%) and nDARC Y41F (100\%) while other parameters maintained $100 \%$.

These preliminarily computational assessments [7-9, 19] are therefore used to demonstrate how a computerized approach (ISM) could be engaged to assess pharmacological activities. Studies carried out on the pharmacological 
activities of these agents with the aim of extracting their sequence information and biological parameters are reported in section 2.1 .

Others computer-based assessments of pharmacological activities have been reported [23-26]. For example, HCV protein sequences associated with the Interferon/Rabavarin combination therapy have been determined using ISM [23]. Suggested targeting of the Extracellular matrix in the designing of therapeutic agents for Ebola Virus was accomplished using computerized procedure (ISM) [5]. Additionally, direct interaction between the beta-adrenoceptors and insulin receptors has been shown using computerized technique (ISM) [24]. Computerized pharmacological assessments achieved using ISM also include the demonstration of the possible mechanism by which Influenza vaccine help protect us from cardiovascular diseases [25]. Over 1000 proteins from 25 functional groups such Oncogenes, Heat Shock Proteins, Protease Inhibitors, SV40 Enhancer, Tumor Necrosis Factors, etc have been assessed using a computerized approach, RRM from which several pharmacologically bioactive peptides (drugs and vaccines, and their candidates) have been designed from [5].

Obermeier et al have devised a computerized procedure and a program [26] that determines susceptibility of HIV strains to a class of antiretroviral agents (CCR5 antagonists) prior to treatment using the $\mathrm{V} 3$ sequence information of the strains. This computer-based pharmacological assessment is demonstrated in this study using two isolates, HXB2 (HIV-1 $\mathrm{T}$ cell lymphocyte-loving virus) and Yu-2 (HIV-1 Macrophage-loving virus).

The procedures taken in the preliminary studies [7-9, 19, 26] are presented in section 3 and the results in section 4 .

\section{Methods}

A Signal Processing technique called Informational Spectrum Method (ISM) and Geno2pheno CORECEPTOR programs are engaged. ISM procedure has been detailed [3-6, 23-25]. A summary of ISM procedure engaged in these studies is provided in section 2.1. The procedure for the Geno2pheno CORECEPTOR program use in this study has been described [27]. The program is available at [http://coreceptor.bioinf.mpi-inf.mpg.de/].

\subsection{Materials}

The materials include:

a. The sequence information of the pharmacologically active agents, their target protein and genes/proteins expressing their activities (four classes) engaged. They are retrieved from the UNIPROT database [30] and various literatures. The sequence information engaged is as shown in Figure 1. Sequences of the V3 motifs belonging to HIV-1 HXB2 and Yu-2 isolates are also extracted from [30] and analyzed.

b. All the biological parameters or Amino Acid Scales engaged are as shown in Table 1. They are obtained from [10].
Theprocedures taken to obtain the sequence information and appropriate biological parameters for the four different groups of pharmacological agents are reported as follows:

\subsubsection{Integrase Enzyme Inhibitors (Raltegravir)}

It has been identified that replication of the single strand HIV genome and subsequent maturation is followed by its insertion into the human genome (Integration). This is catalyzed by Integrase Enzyme [7, 28]. The 288 amino-acid length Integrase Enzyme is inhibited by several mechanisms. One such mechanism is the Strand Transfer. Therefore, a Strand Transfer-based biological parameter with a descriptor name LIFS790103 is engaged in the investigation.

For the sequence information, Menendez-Arias has reported association between resistance in the Raltegravir-managed patients with E92Q, F121Y, E138A, G140A, Y143R, S147G, Q148H and N155H mutations [28]. As a result, consensus and susceptible sequences of the HIV Integrase Enzyme are extracted from the UNIPROT [27]. The resistance sequences were subsequently constructed based on mutations mentioned above and analyzed using the computerized approach.

\subsubsection{Fusion/Entry Inhibitors (Enfuvirtide, Sifuvirtide) and Target Protein NHR}

HIV infection starts with binding interaction that occurs between HIV Surface Protein (HIV gp120) and the host CD4 This is followed by a structural re-arrangement in the Transmembrane Protein (HIV gp41) [28]. This structural conformation brings about the CD4 perforation, and subsequently, the transfer of the viral genetic content into the host CD4. Structural re-arrangement is impossible without the formation of Six Helix Bundle (SHB) between the C-terminal of the Heptad Repeat (CHR) and the N-terminal of the Heptad Repeat (NHR) [7, 28]. Both Enfuvirtide, Sifuvirtide are structural analogues of the CHR. Because of the similarity with the CHR, they compete with CHR in interacting with the NHR, an action that inhibit HIV replication. They are therefore called Competitive Antagonists [7].

Sifuvirtide is a product of biomedical engineering of Enfuvirtide, which is achieve through the introduction of Salt Bridge that provided for an increased Helicity. Additionally, replacement of the Glutaminic acid at position 119 with Threonine provided for hydrophobic pocket $[9,28]$. Helicity and Hydrophobicity oriented modifications are understood to be responsible for the increased inhibitory effect on the viruses demonstrated by Sifuvirtide [9, 28]. As a result, biological parameters associated with Hydrophobicity and Helicity were obtained from the database [10] and engaged. They are the three Alpha-Helix related biological parameters (BURA740101, PONP800104 and PRAM900102) and the five biological parameters associated with Hydrophobicity (ARGP820101, ENGD860101, FASG890101, JURD980101 and WOLR790101). 


\subsubsection{Malaria Vaccine Candidate Peptides P18 and P32}

Peptides P18 and P32 are derived from the parent protein Plasmodial Circumsporozoite (CS). CS, a Plasmodial multifunctional protein responsible for the invasion of Mosquito's salivary gland, binding of the sporozites to the host liver and inactivating their cell creation mechanism Plasmodial [29]. Because of these properties, CS is considered a good component for vaccine design. Two peptides derived from CS, P18 and P32 are being studied for possible engagement in the design. Their residues were retrieved from literature [29] and UNIPROT database [30] and examined.

For the biological parameters engaged, information about their mechanism of interaction was obtained. Peptide P18 and P32are recognized to interact with their target protein, Glycosaminoglycan (GAG) using negatively charged carboxyl group [22]. This shows that Charge Transfer-based biological parameters are involved. Charge Transfer is found to engage five AASs (CHAM830107, CHAM830108, FAUJ880111, FAUJ880112 and KLEP840101) [30]. These parameters were then used together with the EIIP to examine and compare their potencies.

\subsubsection{Plasmodial Host Protein $n D A R C$ and Its Mutant (nDARC Y41F)}

The 35 protein residues of the Plasmodial Host Protein called Duffy Antigen Receptor Chemokines (DARC) and designated nDARC, which is derived from the N-terminal 60 protein residues of the DARC were found to block interaction with the Plasmodial protein called the Duffy Binding Protein (DBP) [22]. Single mutation in nDARC at position 41, (nDARCY41F) is found to abrogate the entire inhibitory activities [22]. The protein residues of the nDARC are obtained from literature [22] while the mutant nDARC $\mathrm{Y} 41 \mathrm{~F}$ is then constructed as shown in Figure 1.

Clinically, sulphonation of the amino acid Tyrosine at position 41 is found to play a great role in the binding interaction that existed between DARC and DBP [22]. Sulphonation is found to be governed by bio-functionalities that involve Charge-Transfer, Negative and Positive Charges. Four biological parameters involved in Charge-Transfer, Negative and Positive Charges, namely, CHAM830107, CHAM830108, FAUJ880112, KLEP840101 are acquired from GeomeNet Database Resources (www.genome.jp/aaindex) [10] and engaged. Prior to sulphonation, there is bio-recognition and bio-attachment, which is ruled by EIIP. As a result, EIIP is also employed.

\subsubsection{Geno2pheno [CORECEPTOR]-based Analysis of HIV-1 T-tropic HXB2 and M-tropic Yu-2 Isolates}

The procedure for the engagement of geno2pheno CORECEPTOR-based determination of pharmacological activity, susceptibility of HIV isolates to CCR5 antagonists using their Hyper-variable V3 domain sequences have been described [27] and expressed as geno 2 pheno $_{\mathrm{FPR}=5 \%}$. Two materials are required here. They are the protein sequences of the V3 domain belonging to the HIV-1 HXB2 and Yu-2 as well as the geno2pheno CORECEPTOR program. The sequences are retrieved from UNIPROT [30], while the program is available at [http://coreceptor.bioinf.mpi-inf.mpg.de/].

Table 1. Thirteen Biological parameters(Amino Acid Scales) involved in the Pharmacological assessments.

\begin{tabular}{|c|c|c|c|c|c|c|c|c|c|c|c|c|c|}
\hline Amino Acids & EIIP & $\begin{array}{l}\text { BURA74 } \\
0101\end{array}$ & $\begin{array}{l}\text { PON } \\
\text { P8001 } \\
04 \\
\end{array}$ & $\begin{array}{l}\text { PRA } \\
\text { M9001 } \\
02 \\
\end{array}$ & $\begin{array}{l}\text { ARGP } \\
82010 \\
1 \\
\end{array}$ & $\begin{array}{l}\text { ENGD } \\
860101\end{array}$ & $\begin{array}{l}\text { FAS } \\
\text { G890 } \\
101 \\
\end{array}$ & $\begin{array}{l}\text { JUR } \\
\text { D980 } \\
101 \\
\end{array}$ & $\begin{array}{l}\text { WOL } \\
\text { R7901 } \\
01 \\
\end{array}$ & $\begin{array}{l}\text { СНА } \\
\text { M8301 } \\
07 \\
\end{array}$ & $\begin{array}{l}\text { CHA } \\
\text { M830 } \\
108 \\
\end{array}$ & $\begin{array}{l}\text { FAUJ } \\
88011 \\
2 \\
\end{array}$ & $\begin{array}{l}\text { KLEP } \\
84010 \\
1 \\
\end{array}$ \\
\hline Alanine (A) & 0.0373 & 0.486 & 13.65 & 1.29 & -1.6 & -1.6 & -0.21 & 1.10 & 1.12 & 0.00 & 0.00 & 0.00 & 0.00 \\
\hline Arginine (R) & 0.0959 & 0.262 & 11.28 & 0.96 & 12.3 & 12.3 & 2.11 & -5.10 & -2.55 & 0.00 & 1.00 & 0.00 & 1.00 \\
\hline Asparagine $(\mathrm{N})$ & 0.1263 & 0.193 & 12.24 & 0.90 & 4.8 & 4.8 & 0.96 & -3.50 & -0.83 & 1.00 & 1.00 & 0.00 & 0.00 \\
\hline Aspartic acid (D) & 0.0036 & 0.288 & 10.98 & 1.04 & 9.2 & 9.2 & 1.36 & -3.60 & -0.83 & 1.00 & 0.00 & 1.00 & -1.00 \\
\hline Cysteine (C) & 0.0829 & 0.200 & 14.49 & 1.11 & -2.0 & -2.0 & -6.04 & 2.50 & 0.59 & 0.00 & 1.00 & 0.00 & 0.00 \\
\hline Glutamine (Q) & 0.0761 & 0.418 & 11.30 & 1.27 & 4.1 & 4.1 & 1.52 & -3.68 & -0.78 & 0.00 & 1.00 & 0.00 & 0.00 \\
\hline $\begin{array}{l}\text { Glutamic acid } \\
\text { (E) }\end{array}$ & 0.0058 & 0.538 & 12.55 & 1.44 & 8.2 & 8.2 & 2.30 & -3.20 & -0.92 & 1.00 & 0.00 & 1.00 & -1.00 \\
\hline Glycine (G) & 0.0050 & 0.120 & 15.36 & 0.56 & -1.0 & -1.0 & 0.00 & -0.64 & 1.20 & 1.00 & 0.00 & 0.00 & 0.00 \\
\hline Histidine $(\mathrm{H})$ & 0.0242 & 0.400 & 11.59 & 1.22 & 3.0 & 3.0 & -1.23 & -3.20 & -0.93 & 0.00 & 1.00 & 0.00 & 0.00 \\
\hline Isoleucine (I) & 0.0000 & 0.370 & 14.63 & 0.97 & -3.1 & -3.1 & -4.81 & 4.50 & 1.16 & 0.00 & 0.00 & 0.00 & 0.00 \\
\hline Lysine (K) & 0.0371 & 0.402 & 11.96 & 1.23 & 8.8 & 8.8 & 3.88 & -4.11 & -0.80 & 0.00 & 1.00 & 0.00 & 1.00 \\
\hline Methionine (M) & 0.0823 & 0.417 & 13.40 & 1.47 & -3.4 & -3.4 & -3.66 & 1.90 & 0.55 & 0.00 & 1.00 & 0.00 & 0.00 \\
\hline $\begin{array}{l}\text { Phenylalanine } \\
\text { (F) }\end{array}$ & 0.0946 & 0.318 & 14.08 & 1.07 & -3.7 & -3.7 & -4.65 & 2.80 & 0.67 & 0.00 & 1.00 & 0.00 & 0.00 \\
\hline Proline $(\mathrm{P})$ & 0.0198 & 0.208 & 11.51 & 0.52 & 0.2 & 0.2 & 0.75 & -1.90 & 0.54 & 0.00 & 0.00 & 0.00 & 0.00 \\
\hline Serine (S) & 0.0829 & 0.200 & 11.26 & 0.82 & -0.6 & -0.6 & 1.74 & -0.50 & -0.05 & 0.00 & 0.00 & 0.00 & 0.00 \\
\hline Threonine (T) & 0.0941 & 0.272 & 13.00 & 0.82 & -1.2 & -1.2 & 0.78 & -0.70 & -0.02 & 0.00 & 0.00 & 0.00 & 0.00 \\
\hline Tryptophan (W) & 0.0548 & 0.462 & 12.06 & 0.99 & -1.9 & -1.9 & -3.32 & -0.46 & -0.19 & 0.00 & 1.00 & 0.00 & 0.00 \\
\hline Tyrosine (Y) & 0.0516 & 0.161 & 12.64 & 0.72 & 0.7 & 0.7 & -1.01 & -1.3 & -0.23 & 0.00 & 1.00 & 0.00 & 0.00 \\
\hline Valine (V) & 0.0057 & 0.379 & 12.88 & 0.91 & -2.6 & -2.6 & -3.50 & 4.2 & 1.13 & 0.00 & 0.00 & 0.00 & 0.00 \\
\hline
\end{tabular}




\subsection{Experimental Procedure}

Informational Spectrum Method (ISM) [3-5, 13, 23, 25], a Physio-mathematical approach was engaged. ISM procedure is provided below.

\subsubsection{Informational Spectrum Method(ISM)-Procedures}

Pharmacological interactions between the interacting components (pharmacological agents and their target proteins) entail two sets of interaction. Pharmacological agents may be the peptidic or protein-based drugs and vaccines, their protein targets or genes/proteins expressing them. First is the binding interaction (bio-recognition and bio-attachment), which is regarded as Inter-molecular interaction. It is found to occur at the distance range greater than 100 Armstrong [5]. This is followed by other interactions that takes place at a closer range and involves structural and/or physiochemical properties. This is called Intra-molecular interaction. ISM assesses both interactions using appropriate biological parameters (Amino Acid Scales). In the case of Inter-molecular interaction, only one biological parameter (Amino Acid Scale) called EIIP is involved. Evaluations of Intra-molecular interaction, which embody structural and physiochemical assessments employ all others biological parameters. It has been recognized that there are over 565 biological parameters (AAs) [14] indicating various modes of bio-molecular interactions in the body. Each scale is for a particular interaction embodying numerous interactions in the body ranging from the simple enzymatic digestion of various food components (proteins, carbohydrates, etc) to more complex bio-transformations carried out by the liver.

The two basic steps involved in molecular interaction have been explained [5]. They are briefly discussed.

\section{Peptide Identity Amino acid sequences and their alignment \\ T20 \\ Sifuvirtide \\ YTSLIHSLIEESQNQQEKNEQELLELDKWASLWNWF SWETWEREIENYTRQIYRILEESQEQQDRNERDLLE \\ P18 \\ P32 IEQYLKKIKNSISTEWSPCSVTCGNGIQVRIK \\ EWSPCSVTCGNGIQVRIK \\ nDARC AELSPSTENSSQLDFEDVWNSSYGVNDSFPDGDYD nDARC Y 41F AELSPSTENSSQLDFEDVWNSSYGVNDSFPDGDFD}

Figure 1. Showing the amino acid sequences of the pharmacological agents engaged. They are nDARC and the constructed $n D A R C Y 41 F$ sequence, Enfuvirtide, Sifuvirtide, and Peptides P18 and P32.

\subsubsection{Inter-molecular Interactions}

Inter-molecular interaction is the first steps involved in molecular interaction [5,9]. At a distance longer than 2-3 Armstrong, bio-molecules interact under the influence of long range interacting force that necessitates productive collusion (Bio-recognition and Bio-attachment). The long range interacting forces is governed by the Valence Electrons and Electron Ion Interaction Potential (EIIP). This has been defined as energy in terms of valence electrons [3-6, 13, 2325]. In case of bio-molecules including the molecules of pharmacological agents and their molecular targets such as proteins and peptides or genes and proteins encoding them, their EIIP values have preliminarily been determined [3-6] using the equation obtained from the General Model Pseudo-Potential [3-6] and shown below.

$$
\mathrm{W}=0.25 Z^{*} \sin \left(1.04 \pi Z^{*}\right) 2 \pi
$$

$\mathrm{Z}^{*}$ is considered as the average quasivalence number $(A Q V N)$ derived through an equation

$$
\mathrm{Z}^{*}=\Sigma \mathrm{mniZi} / \mathrm{N}
$$

$\mathrm{Zi}$ represents the valence number of the $\mathrm{i}^{\text {th }}$ atomic constituent, while ni is the number of atoms of the $i^{- \text {th }}$ constituent. ' $m$ ' denotes the number of atomic constituents in the molecule. $\mathrm{N}$ remains the total number of atoms. EIIP values are expressed in Rydbergs (Ry).

EIIP rules the first interaction (Inter-molecular) and engages the three following processes:

\section{(i). Interchanging of the Alphabetic Codes of the Protein Sequences into Numerical Sequences (Signals) Using EIIP Values}

The amino sequences of the pharmacological agents (where they are protein- or peptide-based), and their protein targets are first converted into numerical sequences using the EIIP values obtained from [10]. The numerical sequences, also called signals represent the behavior or property of the protein in terms of binding with other proteins.

They are then zero-padded. This implies adding zero to the short sequence length until all the sequences share same window size/length. It has been determined that zero-padding is of no biological significance in the analysis [3-6, 9].

\section{(ii). Processing of the Numerical Sequences (Signals) Using Discrete Fourier Transform (DFT)}

The numerical sequences (signals) of pharmaceutical agents (which may be the genes/proteins expressing the 
pharmacological activities or their protein targets) are then processed using Discrete Fourier Transform (DFT). The processed values are then plotted to obtain a mirror image, where the first half represents the embedded pharmacological behavior of the drugs. The mirror is a characteristic of Fourier Transform procedure engaged. Additionally, according to the procedure, the first and/or last values are considered invalid and termed Direct Current (DC) [31]. Simplified version of the ISM-based approach to analyzing proteins using Fourier Transform procedures for non-Engineers has been provided [9] and at [www.biomedfrontiers.org/infection-2014-4-6].

Discrete Fourier Transform is expressed as:

$$
X(n)=\sum x(m) e^{-j(2 / N) n m}
$$

$\mathrm{n}$ represents discrete time (unprocessed) integers from 0,1 , $2, \ldots \mathrm{N}-1 ;(\mathrm{m})$ signifies the $\mathrm{m}^{\text {-th }}$ member of the numerical sequence; while $\mathrm{N}$ is the length of the numerical series and in the case of protein analysis, sequence length. $\mathrm{N}$ runs from $0-\mathrm{N} / 2$. This is because; $\mathrm{N}$ is halved since the output of the DFT processing is a mirror (symmetric) image. For example, in the analysis of protein residues of $\mathrm{P} 32$ and $\mathrm{P} 18$, where $\mathrm{N}$ is 32 , the IS characteristics presented N/2 as 16 values (running from 0-15 in case of Mathlab program or 1-16 using Python) [19]. In the case of CD4 residues and HIV gp120 where $\mathrm{N}$ is 482 and 508, the IS characteristics showed 240 and 253 respectively [31], excluding the first and last values called DC.

$\mathrm{X}(\mathrm{n})$ is the DFT coefficient or function of the Informational Spectrum (IS) characteristics or behavior, which helps put the pharmacological interactions in a form of amplitudes (on the $\mathrm{y}$-axis), and frequencies or positions (on the x-axis).

The expressed behavior is called the Informational Spectrum characteristics. In this manner, the pharmacological behaviors of the pharmaceutical agents, which may be protein/peptide-based drugs/vaccines, or their protein targets or the genes expressing them, are presented in numerical terms (quantified). Spectral characteristic is represented as:

$$
\mathrm{S}(\mathrm{n})=\mathrm{X}(\mathrm{n}) \mathrm{X}^{*}(\mathrm{n})=|\mathrm{X}(\mathrm{n})| 2
$$

Superscript is an indication that the outcomes are conjugate components [31]. This shows that there are real, imaginary and absolute values. Absolute values are engaged. It is important to note that in the ISM analysis, protein residues are presumed to be at equal distance from one another (equidistant) and with $\mathrm{d}=1$.

Maximum frequency therefore can be obtained as:

$$
\mathrm{F}=1 / 2 \mathrm{~d}=0.5
$$

\subsubsection{Common Informational Spectrum}

In any pharmacological action or any other biological activity where more than one protein sequence are found to share common behavior, they are known to demonstrate consensus position of interaction. For example, the CD4 of human, mouse, monkeys, chimpanzee, and other mammals demonstrate common functionality in terms of binding to the Surface Protein (gp120) of several HIV isolates. Similarly,
Enfuvirtide and Sifuvirtide interact with a common HIV target protein, NHR. Therefore, Point-Wise Multiplication is needed to access their point of interaction called Consensus Frequency (CF), and their various contributions in the interaction. This process is called Common Informational Spectrum (CIS).

Mathematically, Point-Wise Multiplication of the Informational Spectra is defined as:

$$
C(j)=\Pi S(j)
$$

$\mathrm{S}(\mathrm{j})$ represents the power spectrum of the $\mathrm{j}^{- \text {th }}$ component.

\subsubsection{Intra-molecular Interactions}

This is the second part of the interaction and it involves all the structural and physio-chemical interactions engaged. After binding with the target proteins, pharmacological agents interact with them intra-molecularly. The interaction may be structural using Helix or Alpha structural arrangement or physio-chemical in which, properties like Hydrophobicity, Ampiphilicty are engaged. It is necessary to study the mechanisms of interaction in order to help ascertain the biological parameters engaged

Above described procedure is employed using each biological parameter and mutations involved in the intra-molecular interaction. The values are then aggregated to obtain complete pharmacological activities. These procedures have been engaged in calculating bio-functionalities [19].

\section{Results}

The positions of common binding interactions between the pharmacologically active agents and their target proteins or genes/proteins expressing them called Consensus Frequency (CF) is first determined and engaged in verifying the contributions by all the interacting components.

$\mathrm{CF}$ is defined as:

$$
\mathrm{CF}=\mathrm{MPP} / \mathrm{N}
$$

MPP is the Maximum Peak Position while N stands for the sequence window size or length.

For example, the CF of the analysis of P18 and P32 where N (sequence sequence window size) is 32 and the Maximum Peak Position using EIIP (Table 3) is 12 can be calculated as;

$$
\mathrm{CF}=12 / 32=0.375
$$

Similarly, using CHAM830107 (Figure 4A), CF can be obtained as $3 / 32$, which is 0.09375 . These results are as shown in Table 3.

The figures presented in this research have preliminarily been shown $[9,19]$. These figures can be accessed from the researches.

Computerized ISM-based findings preliminarily obtained for four classes of drugs [7-9, 19] are presented in this section in order to help demonstrate how these approaches can effectively be employed in decoding pharmacological properties pharmacological investigations. The findings 
presented came from computerized assessments of resistance offered by one antiretroviral agent, HIV Integrase Enzyme Inhibitors (Raltegravir). Results of investigations into resistance offered by others four categories of the antiretroviral agents have been reported [8, 9]. Other outcomes presented here include the comparison of the efficacies of two HIV Fusion/Entry Inhibitors, Enfuvirtide and Sifuvirtide, as well as Malaria vaccine peptide candidates, P18 and P32 [9, 19]. The results of the abrogation of inhibitory effect of the Plasmodial Host Protein nDARC by its mutant (nDARC Y41F) [19] are also presented. Finally, the outcomes of the geno2-pheno CORECEPTOR-based analyses [26] of two HIV-1 strains, HXB2 and Yu-2 are also shown. These are used to show how pharmacological activities can be obtained using computerized procedures. The results are presented in the following sub-sections.

\subsection{Computerized Assessments of the Resistance Offered by Antiretroviral Agents: HIV Integrase Enzyme Inhibitors (Raltegravir) as an Example}

We have preliminarily shown that pharmacological investigations can be carried out using computerized DSP-based procedures like ISM and RRM [8, 9]. Using one biological parameter only, five classes of HIV/AIDS drugs were used to show how pharmacological activities could be investigated $[8,9]$. Here, the results of one group, the Integrase Enzyme Inhibitors (Raltegravir) are presented.

Figure 2 shows the results of the resistance offered by Raltegravir, an Integrase Enzyme Inhibitor using a biological parameter with descriptor called LIFS790103. The results show that at the Consensus Frequency (position 67); the sequence of the resistant strain displayed a reduced level of activity (96.84\%) unlike the susceptible isolate (100\%).

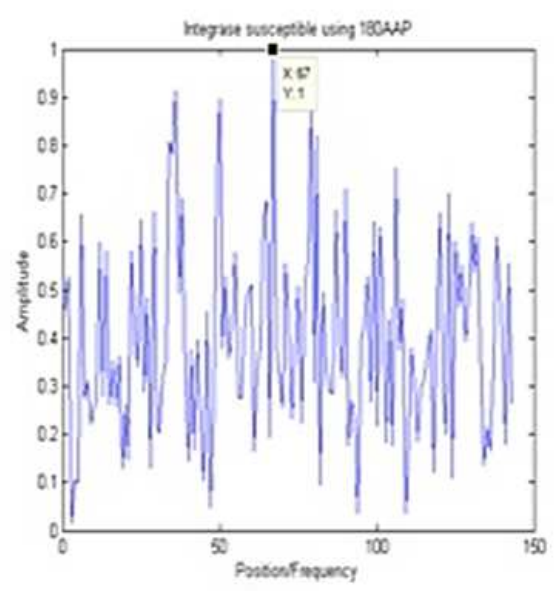

A

Common Informational Pectrum of Susceptible and resistance sequences

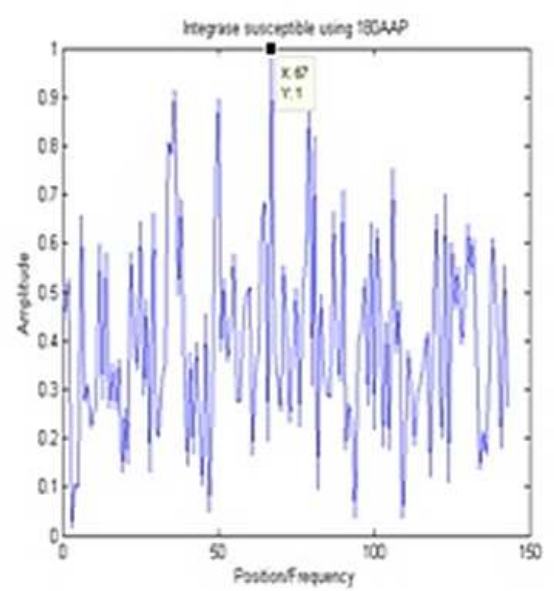

B

Informational Spctrum of the Susceptible sequence

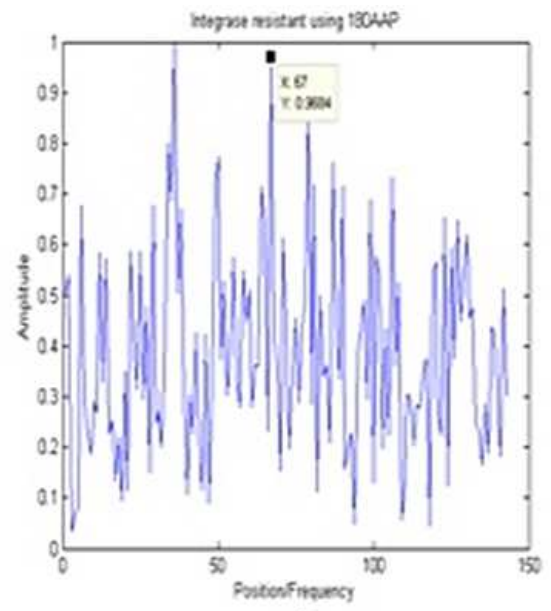

C

Informational Spectrum of the Resistant sequence

Figure 2. Showing Figure $2 A$ as the Common Informational Spectrum (CIS) or Point-wise Multiplication of the Informational Spectrum (IS) of the susceptible and resistant sequences of Integrase Enzyme Inhibitors exposed to antiretroviral agent, Raltegravir; Figure $2 B$ is the IS of the susceptible sequences presenting $100 \%$ interaction; while Figure $2 \mathrm{C}$ is the IS of the resistant sequences displaying 96.84\% activity using one parameter, LIFS790103, Courtesy of [9].This indicates that resistant strains provided less contribution, which is in accord with the clinical findings.

\subsection{Computerized Comparison of HIV Fusion Inhibitors (Enfuvirtide and Sifuvirtide) and Target Protein Using all the Biological Parameters Engaged}

Here, all the nine biological parameters engaged by the HIV Fusion Inhibitors and Target Protein are employed.They areEIIP, BURA740101, PONP800104, PRAM900102, ARGP820101, ENGD860101, FASG890101, JURD980101 and WOLR790101.The outcomes are then aggregated.
The results of Common Informational Spectra (CIS) using three of the parameters (EIIP, ARGP820101 and PONP800104) are as shown in Figure 3. Table 2 presents the entire results. As shown in Table 2, Sifuvirtide provided greater interaction $(85.42 \%)$ than the Enfuvirtide $(72.33 \%)$ on the target protein, NHR, which offered $87.07 \%$. It is observed that anti-retroviral agent Sifuvirtide out-performed Enfuvirtide in all other parameters except WOLR790101. 


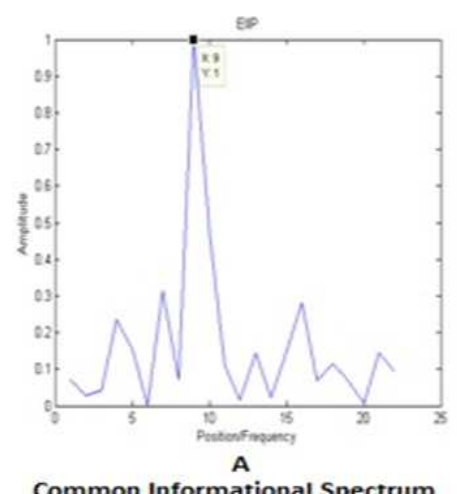

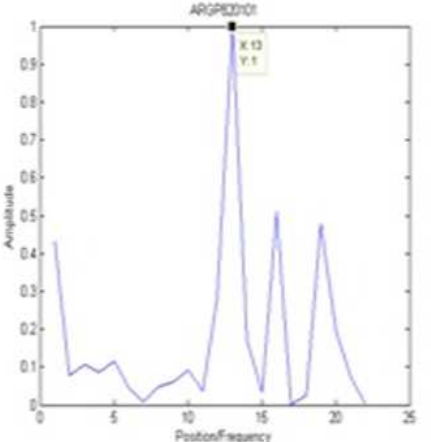

Common Informational Spectrum

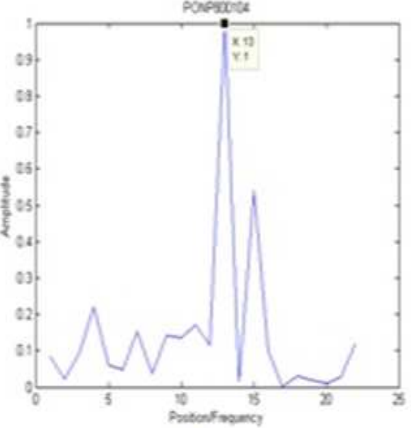

Figure 3. Showing Figure $3 A$ as the Common Informational Spectrum (CIS) or Point-wise Multiplication of the Informational Spectrum (IS) of Enfuvirtide, Sifuvirtide, and NHR using three of the nine biological parameters engaged namely EIIP; Figure 3B for biological parameter, ARGP820101 and Figure 3C, for PONP800104, Courtesy of [9, 19]. The results indicate that each biological parameter is capable of displaying Consensus Frequency or Point of Common Interaction by means of the anti-retroviral agents and their target protein.

Table 2. Showing result of the entire pharmacological assessment and comparison of the efficacies of the two HIV Fusion/Entry Inhibitors Enfuvirtide, Sifuvirtide and their target protein, NHR using the nine parameters engaged, Courtesy of [9, 19].The result correlated with the clinical claim, which stated that Sifuvirtide is more efficacious.

\begin{tabular}{|c|c|c|c|c|c|c|c|c|c|c|}
\hline Amino Acids & EIIP & $\begin{array}{l}\text { BURA740 } \\
101\end{array}$ & $\begin{array}{l}\text { PONP80 } \\
0104\end{array}$ & $\begin{array}{l}\text { PRAM90 } \\
0102\end{array}$ & $\begin{array}{l}\text { ARGP8 } \\
20101 \\
\end{array}$ & $\begin{array}{l}\text { ENGD860 } \\
101\end{array}$ & $\begin{array}{l}\text { FASG8 } \\
90101 \\
\end{array}$ & $\begin{array}{l}\text { JURD9 } \\
80101 \\
\end{array}$ & $\begin{array}{l}\text { WOLR } \\
790101 \\
\end{array}$ & $\begin{array}{l}\text { Average } \\
\text { Interaction }\end{array}$ \\
\hline Enfuvirtide & $81.5 \%$ & $46.7 \%$ & $74.5 \%$ & $64.80 \%$ & $81.0 \%$ & $63.7 \%$ & $84.1 \%$ & $82.8 \%$ & $80.0 \%$ & $72.33 \%$ \\
\hline Sifuvirtide & $100 \%$ & $63.9 \%$ & $76.4 \%$ & $78.5 \%$ & $100 \%$ & $85.1 \%$ & $88.5 \%$ & $97.1 \%$ & $79.5 \%$ & $85.42 \%$ \\
\hline NHR & $77.8 \%$ & $47.9 \%$ & $100 \%$ & $82.1 \%$ & $91.5 \%$ & $90.7 \%$ & $100 \%$ & $98.6 \%$ & $95.0 \%$ & $87.07 \%$ \\
\hline Consensus Frequency & 0.281 & 0.562 & 0.283 & 0.283 & 0.283 & 0.283 & 0.283 & 0.283 & 0.283 & \\
\hline Position & 9 & 18 & 13 & 13 & 13 & 13 & 13 & 13 & 13 & \\
\hline
\end{tabular}

\subsection{Computerized Assessment of Potencies of Malaria Vaccine Candidates P18 and P32 Using all the Biological Parameters Engaged}

Table 3 shows the computer-based results generated using EIIP and five Charge Transfer-based biological parameters involved in the interaction.Charge Transfer, which involves negatively charged carboxyl group, is found to be the major mode of interaction between the peptides P18 and P32 and the target protein Glycosaminoglycan (GAG) [22]. The parameters are namely, CHAM830107, CHAM830108, FAUJ880111, FAUJ880112, and KLEP840101. Figure 4 presents the results derived by means of one biological parameter, CHAM830107. Both peptides are found to maintain maximum affinity (binding interaction) of $100 \%$ by means of EIIP. On the whole, Peptide P32 offered greater interaction (95.15\%) than peptide P18 (95.15\% 93.40\%). The point of common interaction (Consensus Frequency, CF) is at $\mathrm{f}=0.375$ or position 3 .

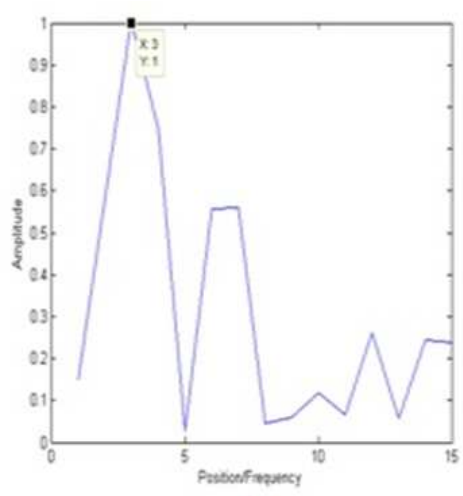

A

Common Informational Spectrum

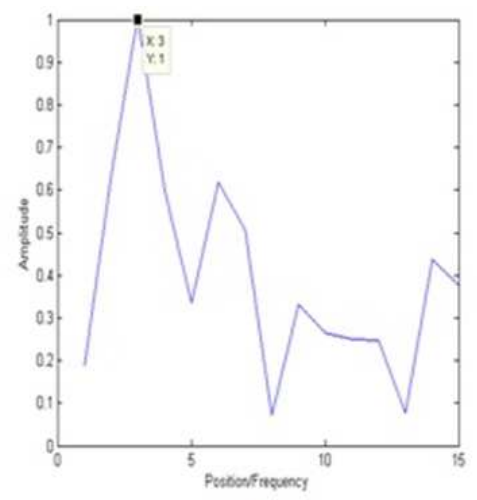

Informational Spectrum of P18

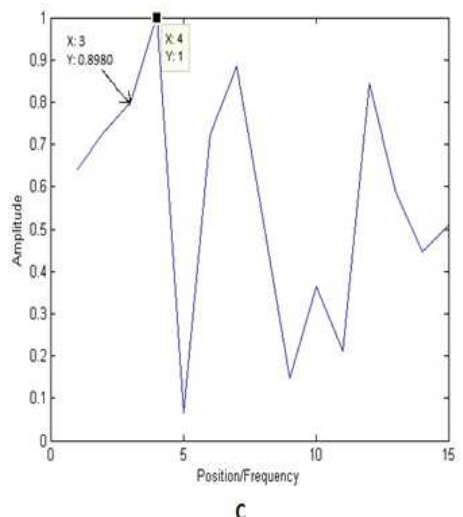

Informational Spectrum of P32

Figure 4.Showing Figure $4 A$ as the Common Informational Spectrum (CIS) or Point-wise Multiplication of the Informational Spectra (IS) of peptidesP18 and $P 32$ as well as the Consensus Frequency (CF=3); Figure $4 B$ as the Informational Spectrum (IS) of P18, showing amplitude of 1.0 at CF=3; and Figure 4C as the Informational Spectrum (IS) of P32 displaying amplitude of 0.8980 at CF=3. The results are obtained using CHAM830107, Courtesy of [9, 19]. This indicates one of the decreases in the amplitude (level of interaction) by the P18 that gave P32 an advantage in the overall contribution. 
Table 3. Showing result of the entire pharmacological assessment and comparison of the efficacies of the two Malaria vaccine candidates peptide P18 andP32 using the six parameters engaged, EIIP, CHAM830107, CHAM830108, FAUJ880111, FAUJ880112 and KLEP840101, courtesy of [9, 19].

\begin{tabular}{llllllll}
\hline Amino Acids & EIIP & CHAM830107 & CHAM830108 & FAUJ880111 & FAUJ880112 & KLEP840101 & Average Interaction \\
\hline P18 & $100 \%$ & $100 \%$ & $100 \%$ & $100 \%$ & $71.6 \%$ & $88.80 \%$ & $93.40 \%$ \\
P32 & $100 \%$ & $89.80 \%$ & $81.10 \%$ & $100 \%$ & $100 \%$ & $100 \%$ & $95.15 \%$ \\
Consensus Frequency & 0.375 & 0.094 & 0.438 & 0.032 & 0.156 & 0.094 & \\
Position & 12 & 3 & 14 & 1 & 5 & 3 & \\
\hline
\end{tabular}

\subsection{Computerized Assessment of the Single}

Mutation-Induced Abolition of the Inhibitory Activity of the Plasmodial Host Protein NDARC on DBP Using all the Biological Parameters Engaged

The outcomes of computerized based analyses of the abolition of inhibitory activity of the nDARC by a single mutation nDARC Y41F are as shown in both Table 4 and Figure 5. Table 4 shows the entire findings while Figure 5 presents only the EIIP-based results indicating the amplitude of 0.844 (i.e. $84.4 \%$ activity) for the $\mathrm{nDARC}$ and an increased in amplitude of 1.00 (i.e. $100 \%$ activity) for the mutant nDARC Y41F.

Table 4. Results of the ISM-based Pharmacological investigations on nDARC and mutant nDARC Y4IF using the five of the pharmacological parameters (EIIP, CHAM830107, CHAM830108, FAUJ880112 and KLEP840101) engaged indicating the abrogation (abolition) of the inhibitory effect of the nDARC (96.88\%) by the introduction of a single mutation Y4IF (100\%), courtesy of [9]. EIIP is the only source of change in the amplitude indicating binding interaction as the major mechanism of action.

\begin{tabular}{llllll}
\hline Amino Acids & EIIP & CHAM830107 & CHAM830108 & FAUJ880112 & KLEP840101 \\
\hline nDARC & $84.4 \%$ & $100 \%$ & $100 \%$ & $100 \%$ & $100 \%$ \\
nDARC Y41F & $100 \%$ & $100 \%$ & $100 \%$ & $100 \%$ & $100 \%$ \\
Consensus Frequency & 0.17 & 0.17 & 0.29 & 0.06 & 0.06 \\
Position & 6 & 6 & 10 & 2 & 2 \\
\hline
\end{tabular}

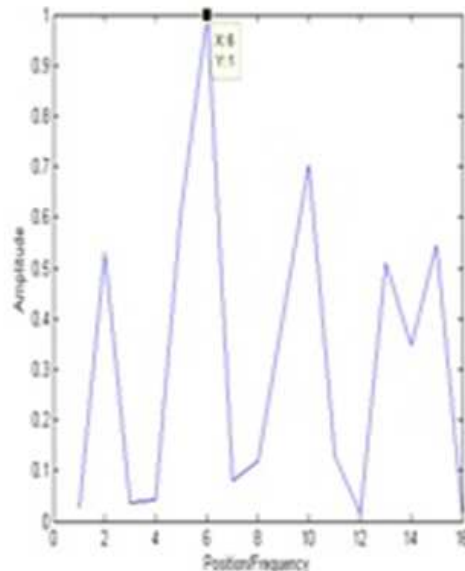

A

Common Informational Spectrum

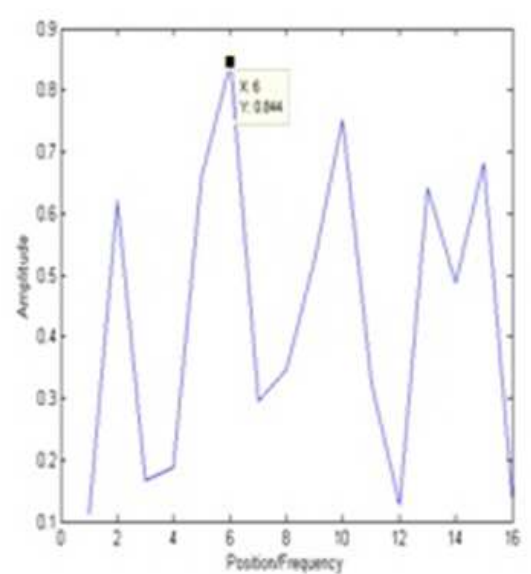

B

Informational Spectrum of nDARC

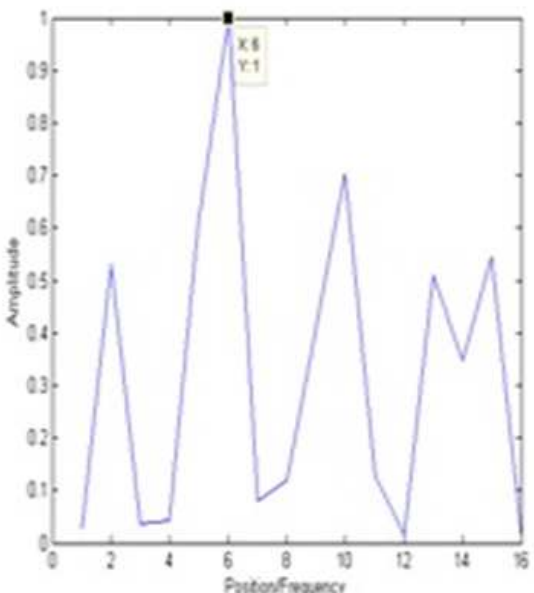

C

Informational Spectrum of nDARC Y41F

Figure 5. Showing 5Aas the Common Informational Spectrum (CIS) or Point-wise Multiplication of the Informational Spectra (IS) of nDARC and nDARC $Y 41 F ; 5 B$ as the Informational Spectrum (IS) of nDARC; and $5 C$ the Informational Spectrum (IS) of $n D A R C$ Y $41 F$, respectively using EIIP. The results displayed the differences in amplitude (level of interactions) between the $n D A R C(96.88 \%)$ and the mutant $n D A R C Y 41 F(100 \%)$. This is an indication of the abolition of the inhibitory effect due to single mutation, courtesy of [9].

\section{Discussions}

The results obtained in Section 3 are discussed in the following subsections:

\subsection{Computerized Assessments Using Antiretroviral Agents}

Computerized assessment of five classes of antiretroviral studied using one biological parameter demonstrated that mutated sequences displayed lower amplitude at the 
positions of common interaction (Consensus Frequency). This signifies that the mutated sequences provided lower level of interaction/contribution than the susceptible sequences [8, 9]. This is evident in Figure 2, which shows that at the Consensus Frequency $(\mathrm{CF}=67)$, the susceptible sequences of Integrase Enzyme Inhibitors exposed to antiretroviral agent, Raltegravir presented 100\% interaction while the resistant sequences displayed $96.84 \%$ activity, respectively using LIFS790103.

This appears to signify that one of the ways the virus could cause resistances to the pharmacological agents they were exposed is through structural modification. HIV exposed to Integrase Enzyme Inhibitors, Raltegravir has shown modification in its Strand structure [28]. As a result, Strand-related parameter called LIFS790103 is engaged in the computerized assessment of resistances offered by the virus to Raltegravir.

Apart from the structural amendment, other forms of modifications involving physio-chemical properties may be engaged. These properties employ physio-chemical parameters such as Hydrophobicity, binding interaction, Ampiphilicity, etc. Maturation Inhibitors (Bevirimat) for example engage binding interaction. In determining the resistance Maturation Enzyme offered to BVM, a binding interaction-based parameter with descriptor name EIIP is employed. These structural and physio-chemical modifications are achieved through alteration of protein sequence alignment (mutations).

Similar results were obtained using sequence information from the other classes of antiretroviral agents, and appropriate biological parameters. The outcomes demonstrated correlation with clinical findings, signifying the reliability of the computerized approaches. For example, Fusion/Entry Inhibitors (Enfurvirtide), which engages Helix conformation-based parameter, designated ROBB760104 showed $100 \%$ contribution for the susceptible sequences and $99.77 \%$ for the resistant sequences. Nucleotide Reverse Transcriptase Inhibitor (Lamivudine), which employs stearic conformation-based parameter described as BEGF750102, offered $100 \%$ for the susceptible sequence and $94.67 \%$ for the resistant sequence. The resistant and susceptible sequences of HIV exposed to Protease Inhibitor (Darunavir) provided $89.95 \%$ and $100 \%$ contribution, respectively when analyzed with Hydrophobicity-oriented parameter, WILM950103 [8, 9].

However, engaging all the parameters and mutations involved will give full pharmacological activity (and in this case, total resistance) as shown in other agents. This is because, it has been identified that at one point mutation, several parameters are employed [14].

\subsection{Computerized Comparison of the Potencies of HIV Fusion Inhibitors and Target Protein Using all the Biological Parameters Engaged}

Both Enfurvirtide (T20) and Sifurvirtide are CHR-based HIV Fusion Inhibitors [20]. Enfurvirtide has been approved by FDA for HIV/AIDS treatment [28]. Sifurvirtide is a product of biomedical engineering, which resulted in increased Helicity and Hydrophobicity. Though Sifurvirtide is yet to be approved for use, it is claimed to be more effective than the Enfurvirtide [20].

Using this computerized approach discussed, this claim was assessed using biological parameters engaged. Three biological parameters relating to Alpha-Helix, BURA740101, PNOP800104 and PRAM9001012 as well as five Hydrophobicity-oriented parameters ARGP820101, ENGD860101, FASG890101, JURD980101, and WOLR790101 are engaged. As shown in Table, results obtained in this study are found to be in accord with the claim. This is because Sifurvirtide offered greater interaction $(85.42 \%)$ than the Enfurvirtide, which provided $72.33 \%$. The target protein for both drugs, the NHR, provided $87.07 \%$. This signifies that Sifurvirtide appear to be more efficacious than the Enfurvirtide. Based on these results, the claim appears to be justified using computerized approach. However, this approach does not provide for, toxicity studies as to ascertain therapeutic indices of the drugs. There are rather, algorithms for toxicity.

\subsection{Computerized Assessment of Potencies of Malaria Vaccine Candidates P18 and P32 Using All the Biological Parameters Engaged}

Another pharmacological activity investigated is the efficacies of the two starter materials for Malaria vaccine design, peptides P18 and P32. Both peptidic candidates are being studied for possible engagement in Malaria intervention. Vaccine potencies are measured based on Specificity and Sensitivity. Specificity defines vaccine's capability to identify correctly, the virus or organism to immunize against. It is concerned with appropriate bio-recognition and bio-attachment (Inter-molecular or binding interaction), which is governed by a biological parameter, EIIP. Based on the computer-derived results (Table 3), both peptides (P18 and P32) demonstrated absolutespecificity as each achieved $100 \%$ affinity with the target protein Glycosaminoglycan (GAG). Intra-molecular interactions, involving other parameters identifies the Sensitivity of the vaccines.

The overall results derived using all the biological parameters engaged indicated high activity for both peptides, P18 (95.15\%) and P32 (93.40\%). This signifies that both peptides are good Malaria vaccine candidates.

\subsection{Computerized Assessment of Abrogatory Effect of Single Mutation (Y41F) on the Plasmodial Host Protein nDARC Using all the Biological Parameters Engaged}

Clinical experimentations have shown that the 35 amino acid length region of the Duffy Antigen Receptor Chemokines (DARC), designated nDARC, which belongs to the Plasmodial hosts (such as human, monkeys, etc) is known to inhibit interaction with the Plasmodial protein called Duffy Binding Protein (DBP). This inhibitory activity is annihilated by a single mutation, Y41F. Sequence 
information as well as the biological parameters engaged in these interactions were obtained and employed in the computer-based assessment of this claim. The results were found to concur with the claim as nDARC's inhibitory activity $(96.88 \%)$ was abolished by the mutant nDARC Y41F, which showed $100 \%$ activity (Table 4 ).

The major mechanism of action, as can be deduced by the computer-based program, appears to have come from bio-recognition and bio-attachment (binding interaction). This activity is controlled by a parameter known as EIIP. This is because; only the EIIP results demonstrated changes in the magnitude of interaction. nDARC is observed to achieved a binding interaction of $84.4 \%$ using EIIP while other parameters gained $100 \%$ interactions. This result is found to concur with the clinical result [22].

\subsection{Computerized Uncovering of Pharmacological Activities as Achieved by Other Researchers}

Obermeier et al had engaged a non-ISM computerized technique to investigate pharmacological properties. They also developed a computerized procedure, geno2pheno CORECEPTOR, which determines susceptibilities and/or resistances of HIV isolates to CCR5 antagonists using their Hyper-variable V3 domain sequences [26]. The program is available at [http://coreceptor.bioinf.mpi-inf.mpg.de/]. The pharmacological assessment carried out in this study was achieved using 5\% False Positive Rate (FPR), a method preliminarily described as geno 2 pheno $_{\mathrm{FPR}=5 \%}$ [24]. Protein sequences of the V3 domain belonging to the HIV-1 HXB2 and Yu-2 are analyzed. The results are as shown in Figures 6 and 7. The outcome of the analysis of the sequence (CTRPNNNTRKSINI**GPGRALYTTGEIIGDIRQAHC) belonging to the $\mathrm{V} 3$ region of the $\mathrm{Yu}-2$ isolate, a Macrophage-loving virus shows that CCR5 antagonist can be administered (Figure 6). This is not the same with the sequence of the T-Cell lymphocyte loving isolate, HXB2 (CTRPNNNTRKSIRIQRGPGRAFVTIGKI*GNMRQAHC

). As shown in Figure 7, this program does not recommend HBX2 treatment with the CCR5 antagonist. These results are consistent with clinical findings [26].

Pharmacological activities of several other agents have been evaluated using computerized procedures. ISM procedures have been engaged in the determination of the $\mathrm{HCV}$ protein sequences responsible for Interferon/Rabavirin combination therapy [15]. Assessment of the impact of the interaction between the Ebola Virus and the host extracellular matirx in the prevention and therapy of the disease [5] was investigated using ISM-based computerized approach. The possible mechanism of action by which Influenza vaccine prevents cardiovascular diseases has computationally been investigated [25].

There are several other pharmacological activities uncovered from several pharmacological agents, proteintargets or genes/proteins expressing them, using computerized approaches, which are not recorded here. Their derived outcomes are found to be in accord with the clinical findings. The computerized procedure employed here is recognized to be simple, fast and resource-saving.
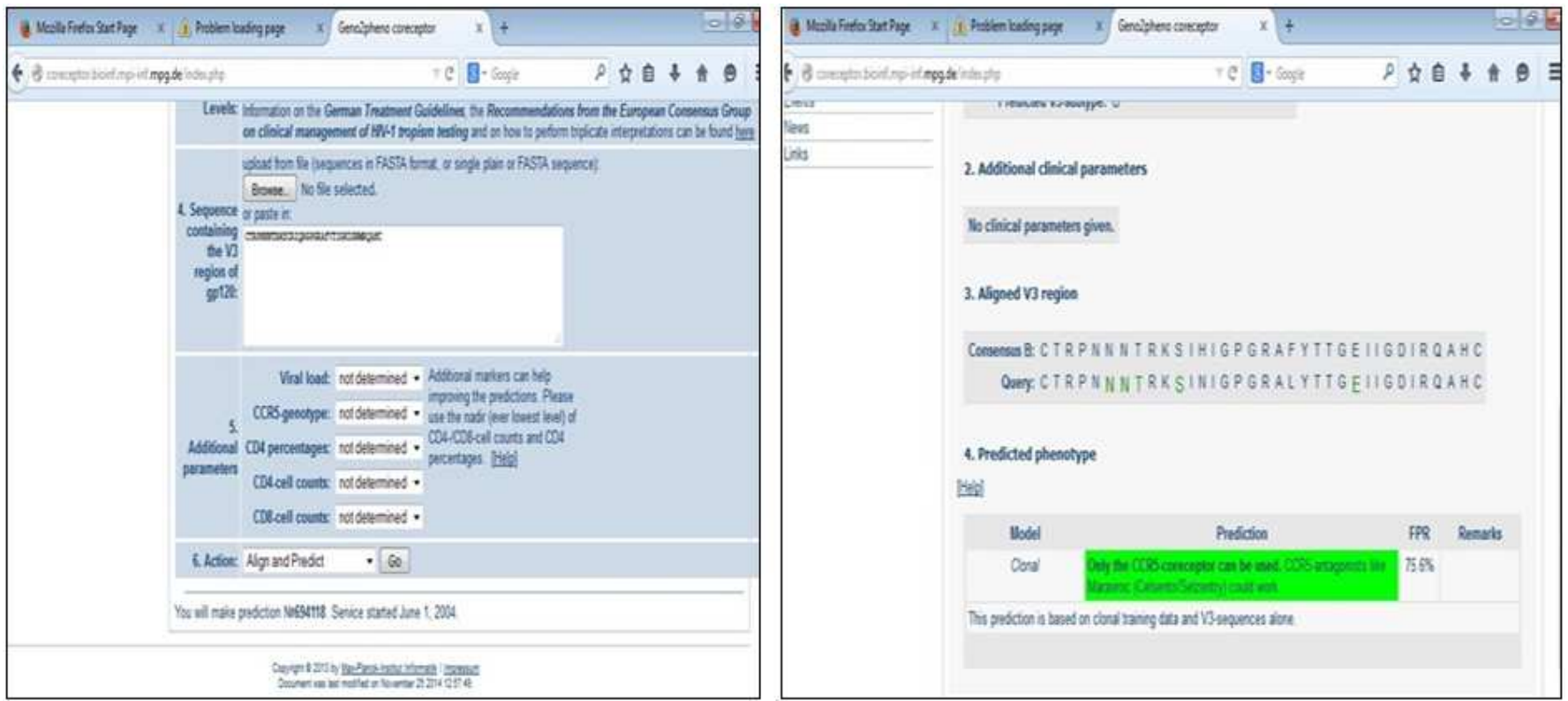

Figure 6. An outcome of the geno2pheno CORECEPTOR program [26] processing of the V3 motif of the belonging to the M-tropic group called Yu-2, symbolizing appropriateness in the administration of CCR5 antagonists to the HIV isolate. 

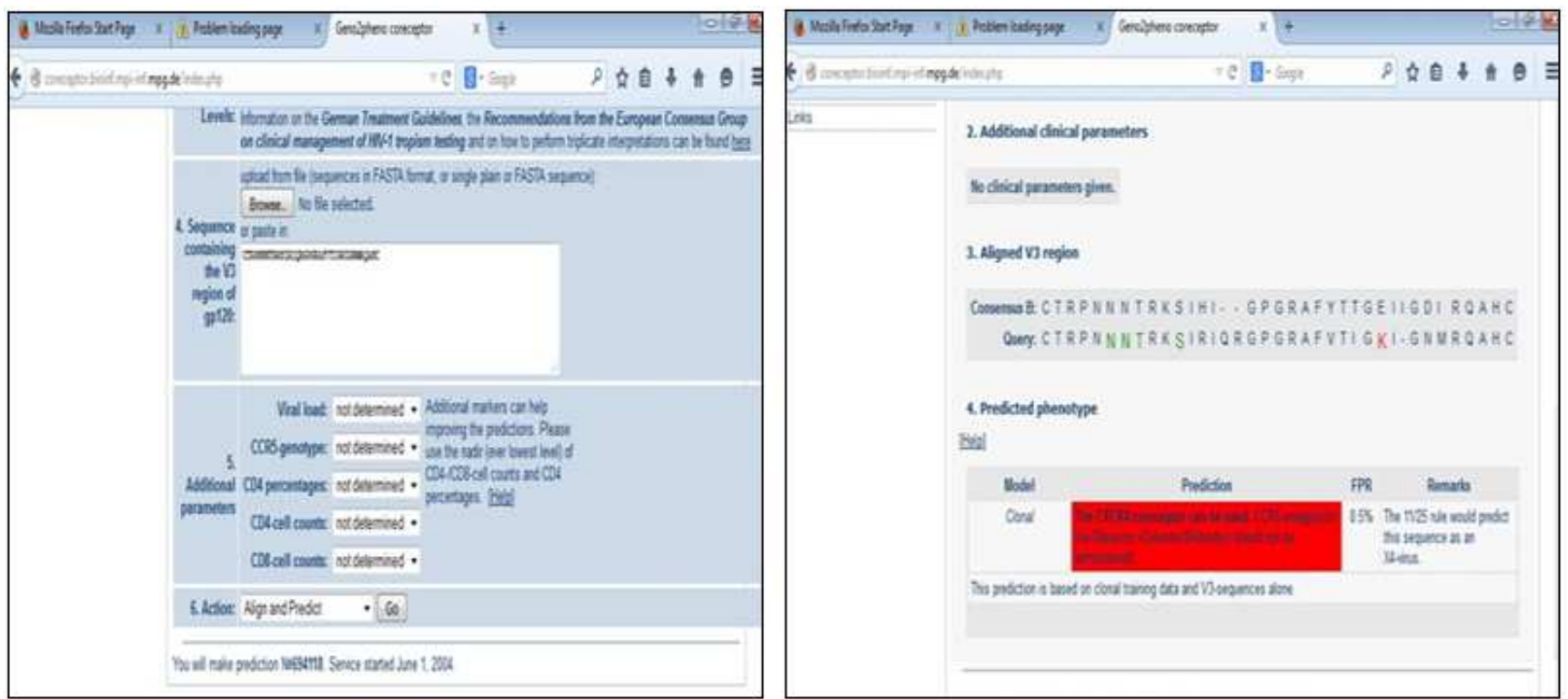

Figure 7. The outcome of the geno2pheno CORECEPTOR program [26] processing of the V3 motif of the belonging to the T-tropic group called HXB2, signifying danger in the administration of CCR5 antagonists to the HIV isolate.

\section{Conclusions}

Currently, we may be complementing clinical assessments with computerized procedures. In future, there may be a complete disengagement from the clinical procedures as biological and pharmacological data such as sequences are increasingly being generated. This signifies the need for computerized procedures in pharmacological studies.

We realized that for effective engagement of ISM, sequence information and biological parameters must be made available and engaged. Genes/proteins are known to provide as much pharmacological information as pharmacological active agents. Pharmacological activities of drugs can now be translated from the sequence information of the agents where they are protein or peptide based. Otherwise, the sequence information of the genes/proteins encoding the agents or their protein targets can be engaged. However, good understanding of the drugs' mode of action at the atomic level is needed since more than one biological parameter may be involved at one point mutation. Cumulative input from each mutation and parameter is therefore required for the totality of pharmacological activity.

By means of all the sequence information and biological parameters we have uncovered series of pharmacological activities from several therapeutic agents using computerized approaches. Several other researchers have records of computerized pharmacological assessments as well. Results obtained clinically and computationally are found to correlate.

Additionally, these approaches are found to have an advantage of presenting pharmacological activities in numerical terms (quantification of the pharmacological characteristics). Based on these, we recommend their engagement in deciphering pharmacological activities of agents where sequence information and biological parameters are available. The procedures and programs engaged in these studies will be of immense assistance to medical professionals and practitioners, researchers, scientists, etc.

Rational, computerized, informatics- and robotics-based procedures are now the revolutionizing factors. They have taken over assessments in most fields such as Architecture, Engineering, etc. Most evaluations in these areas embody computerized, robotics and information technology-based approaches. In this computer and post-genomic era, engaging these procedures in pharmacological investigations and further incorporating them into Pharmaco-informatics programs have become inevitable.

\section{References}

[1] Schmidt B, Ribnicky DM, Pouley A, et al, "A natural history of botanical therapeutics" Metabolism Clinical and Experimental, vol. 57(Suppl 1), pp. S3-S9. 2008.

[2] Finn PW, "Computational approaches to drug design", Algorithmica. Vol. 25(2), pp. 347-371, 1999.

[3] Veljkovic V, Glisic S, Muller P, et al, "Insilico analysis suggests interaction between Ebolavirus and the extracellular matrix", Virology,vol, 6(135), pp.1-11, 2015.

[4] Perovic VR, Muller CP, Niman HL, et al, "Novel Phylogenetic Algorithm to Monitor Human Tropism in Egyptian H5N1-HPAIV Reveals Evolution toward Efficient Human-to-Human Transmission”, PLoS ONE, vol. 8(4), pp. e61572, 2013. doi:10.1371/journal.pone.0061572

[5] Veljkovic V, Veljkovic N, "Characterization of conserved properties of hemagglutinin of $\mathrm{h} 5 \mathrm{n} 1$ and human influenza viruses: possible consequences for therapy and infection control”, BMC Structural Biology, vol. 9(21), pp. 1-11, 2009.

[6] Cosic I, "Macromolecular Bioactivity: Is It Resonant Interaction between Macromolecules?-Theory and Applications," IEEE Transactions on Biomedical Engineering", 41(I):1101-1114, 1994. 
[7] Nwankwo N, "A Digital Signal Processing-based Bioinformatics Approach to Identifying the Origins of HIV-1 non B subtypes infecting US Army Personnel serving abroad", Curr HIV Res, vol. 11(4),pp. 271-80, 2013.

[8] Nwankwo N, Seker H. 2010. "A signal processing-based bioinformatics approach to assessing drug resistance: human immunodeficiency virus as a case study". Conf. Proc. IEEE Eng Med BiolSoc,vol. 2010, pp. 1836-1839.

[9] Nwankwo N, "Signal processing-based Bioinformatics methods for characterization and identification of Bio-functionalities of proteins", $\mathrm{PhD}$ Thesis (accepted), De Montfort University, Leicester, United Kingdom, 2012. (Available at www.openthesis.org).

[10] Tomii K, Kanehisa M, "Analysis of amino acid indices and mutation matrices for sequence comparison and structure prediction of proteins," Protein Engineering, vol. 9(1), pp. 27-36, 1996.

[11] Robson KJH, Naita S, Barker G, Sinden RE, Crisanti A, "Cloning and expression of the thrombospondin-related adhesive protein gene of plasmodium berghei," Molecular and Biochemical Parasitology, vol. 84, pp. 1-12, 1997.

[12] Wilce MC, Aguilar MI, Hearn MT, "Physicochemical basis of amino acid hydrophobicity scales: evaluation of four new scales of amino acid hydrophobicity coefficients derived from RP-HPLC of peptides," J Anal Chem, vol. 67, pp. 1210-1219, 1995.

[13] Veljkovic V. and Veljkovic N, "Characterization of conserved properties of hemagglutinin of $\mathrm{h} 5 \mathrm{n} 1$ and human influenza viruses: possible consequences for therapy and infection control", BMC Structural Biology, vol. 9(21) pp. 1-11, 2009.

[14] Hoj L, Kjaer J, Winther O, Cozzi-Lepri A, Lundgreen DJ, "In silico identification of physicochemical properties at mutating positions relevant to reducing susceptibility to amprenavir," XVII International HIV Drug Resistance Workshop, Poster No.113, 2008.

[15] Forrest MS,Lan Q, Hubbard AE, et al, "Discovery of Novel Biomarkers by Microarray Analysis of Peripheral Blood Mononuclear Cell Gene Expression in Benzene-Exposed Workers".Environmental Health Perspectives, vol. 113(6) pp. 801-807, 2005.

[16] Zhou Y, Gottesman M, Pastan I,"Studies of Human MDR1-MDR2 Chimeras Demonstrate the Functional Exchangeability of a Major Transmembrane Segment of the Multidrug Transporter and PhosphatidylcholineFlippase", Molecular and Cellular Biology, vol. 19(2), pp. 1450-1459, 1999.

[17] International Human Genome Sequencing Consortium, "Finishing the euchromatic sequence of the human genome," NATURE, vol. 431, pp. 931-945, 2004.

[18] Zheng CJ, Han LY, Yap CW, et al, "Therapeutic Targets: Progress of Their Exploration and Investigation of Their Characteristics". Pharmacological Reviews, vol. 58(2), pp. 259-279, 2006.

[19] Nwankwo N, Seker H, "Digital Signal Processing Techniques: Calculating the Biological Functionalities of Proteins" J. Proteomics Bioinform, vol. 4, pp 260-268, 2011. doi:10.4172/jpb.1000199.
[20] He Y, Xiao Y, Song H, Liang Q, Ju D, Chen X, Lu H, Jing W, Jiang S, Zhang L, "Design and evaluation of sifuvirtide, a novel hiv-1 fusion inhibitor," J Biol Chem., vol. 283(17), pp. 11126-11134, 2008.

[21] Chitnis CE, Sharma A, "Targeting the plasmodium vivaxduffy-binding protein," Trends in Parasitology, vol. 24(1), pp. 29-34, 2007.

[22] Pinzon-Ortiz C, Friedman J, Esko J, Sinnis P, "The binding of the circumsporozoite protein to cell surface heparan sulfate proteoglycans is required for plasmodium sporozoite attachment to target cells," J Biol Chem., vol. 276(29), pp. 26784-26791, 2001

[23] Veljkovic V, Glisic S, Veljkovic N, et al, "Assessment of Hepatitis C Virus protein sequences with regard to interferon/ribavirin combination therapy response in patients with HCV genotype 1b", Vaccine, vol 132(48), pp. 6569-6575, 2014.

[24] Mandić M, Drinovec L, Glisic S, "Demonstration of a direct interaction between $\beta 2$-adrenergic receptor and insulin receptor by BRET and bioinformatics. PLoS One, vol. 9(11), pp. e112664, 2014.

[25] Veljkovic V, Glisic S, Veljkovic N, et al. Influenza vaccine as prevention for cardiovascular diseases: Possible molecular mechanism. Vaccine (2014), http://dx.doi.org/10.1016/j.vaccine.2014.07.007

[26] Obermeier M, Ehret R, Berg T, et al, "Genotypic HIV-coreceptor tropism prediction with geno2pheno [CORECEPTOR]: differences depending on HIV-1 subtype“, Journal of the International AIDS Society, vol. 15(Suppl 4), pp.1821-1824, 2012

[27] Secle'n E, Garrido C, Gonzalez MM, "High sensitivity of specific genotypic tools for detection of $\mathrm{X} 4$ variants in antiretroviral-experienced patients suitable to be treated with CCR5 antagonists", AntimicrobChemother, vol.65, pp. 1486-1492, 2010.

[28] Menendez-Arias L, "Molecular basis of Human Immunodeficiency Virus drug resistance: an update," Antiviral Res., vol. 85(1), pp. 210-231, 2010.

[29] Garcia JE, Puentes A, Patarroyo ME, \Developmental biology of sporozoite-host interactions in plasmodium falciparum malaria: implications for vaccine design," ClinMicrobiol Rev., vol. 19(4), pp. 686-707, 2006.

[30] Jain E, Bairoch A, Duvaud S, Phan I, Redaschi N, Suzek BE, Martin MJ, McGarvey P, Gasteiger E,Infrastructure for the life sciences: design and implementation of the UNIPROT website," BMC Bioinformatics, vol. 10, pp. 136-154, 2009.

[31] Smith SW, The Scientist and Engineer's Guide to Digital Signal Processing. California Technical Publishing, 2002.

[32] Nwankwo N, Seker H, "HIV Progression to AIDS: Bioinformatics Approach to Determining the Mechanism of Action". Curr HIV Res,vol. 11(1) pp. 30-42, 2013. 